\title{
Fast and automatic depth control of iterative bone ablation based on optical coherence tomography data
}

Alexander Fuchs, Steffen Pengel, Jan Bergmeier, Lüder A. Kahrs, Tobias Ortmaier

Alexander Fuchs, Steffen Pengel, Jan Bergmeier, Lüder A. Kahrs, Tobias Ortmaier, "Fast and automatic depth control of iterative bone ablation based on optical coherence tomography data," Proc. SPIE 9542, Medical Laser Applications and Laser-Tissue Interactions VII, 95420P (15 July 2015); doi: 10.1117/12.2183695 


\title{
Fast and automatic depth control of iterative bone ablation based on optical coherence tomography data
}

\author{
Alexander Fuchs, Steffen Pengel, Jan Bergmeier, Lüder A. Kahrs, Tobias Ortmaier \\ Institute of Mechatronic Systems, Leibniz Universität Hannover, Hannover, Germany
}

\begin{abstract}
Laser surgery is an established clinical procedure in dental applications, soft tissue ablation, and ophthalmology. The presented experimental set-up for closed-loop control of laser bone ablation addresses a feedback system and enables safe ablation towards anatomical structures that usually would have high risk of damage.This study is based on combined working volumes of optical coherence tomography (OCT) and Er:YAG cutting laser. High level of automation in fast image data processing and tissue treatment enables reproducible results and shortens the time in the operating room. For registration of the two coordinate systems a cross-like incision is ablated with the Er:YAG laser and segmented with OCT in three distances. The resulting Er:YAG coordinate system is reconstructed. A parameter list defines multiple sets of laser parameters including discrete and specific ablation rates as ablation model. The control algorithm uses this model to plan corrective laser paths for each set of laser parameters and dynamically adapts the distance of the laser focus. With this iterative control cycle consisting of image processing, path planning, ablation, and moistening of tissue the target geometry and desired depth are approximated until no further corrective laser paths can be set. The achieved depth stays within the tolerances of the parameter set with the smallest ablation rate. Specimen trials with fresh porcine bone have been conducted to prove the functionality of the developed concept. Flat bottom surfaces and sharp edges of the outline without visual signs of thermal damage verify the feasibility of automated, OCT controlled laser bone ablation with minimal process time.
\end{abstract}

Keywords: automatic bone cutting, ablation control, Er:YAG laser, OCT registration

\section{INTRODUCTION}

Laser surgery is an established clinical procedure, e.g. in ophtalmology or soft tissue interventions. Advantages are contact-free processing, arbitrary geometries, and high precision due to small focus diameters. Up to now, hard tissue ablation is limited mainly to hand held dental applications. Recent developments illustrate a reactivation of pulsed Er:YAG laser sources that present even higher absorption coefficients of water molecules due to its wavelength of $\lambda=2940 \mathrm{~nm}$ promising less heat input in surrounding structures. ${ }^{1,2}$ However, positive effects on bone healing are shown in various studies, if laser parameters are chosen correctly ${ }^{3,4}$ and potential for a more widespread use of laser bone processing is apparent. In most applications, $\mathrm{CO}_{2}$ laser sources are used owing to their high tissue ablation rates.

In order to safely ablate structures containing critical boundaries, e.g. the dura mater at the skull, a feedback system for current ablation depth is missing in most laser systems. For several years different research groups have tried to implement additional sensory information to overcome this problem. ${ }^{5,6,7}$ In this context, optical coherence tomography (OCT) illustrates promising potential, not only regarding its high resolution of only a few micrometers, but also because of its capability of analyzing subcutaneous structures. ${ }^{8}$ In this paper, we present algorithms and experimental results for our prototype ${ }^{9}$ demonstrating closed-loop control of laser bone ablation using OCT data. Providing a high degree of automation (e.g. in necessary preparatory steps and in the actual tissue processing) as well as fast automatic image data computing and transfer we guarantee safe results and short time in the operating room.

Further author information: Alexander Fuchs: E-mail: alexander.fuchs@imes.uni-hannover.de 


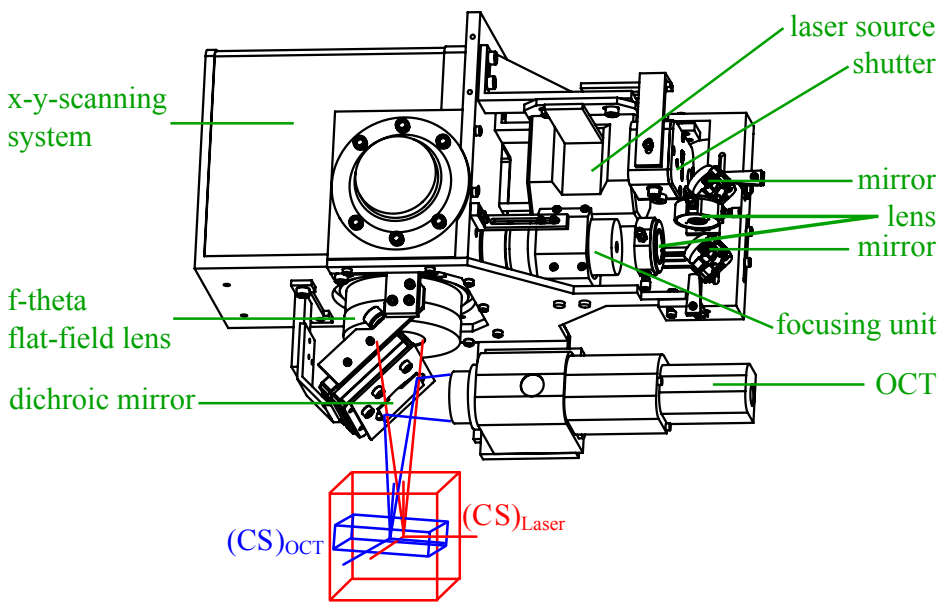

(a)

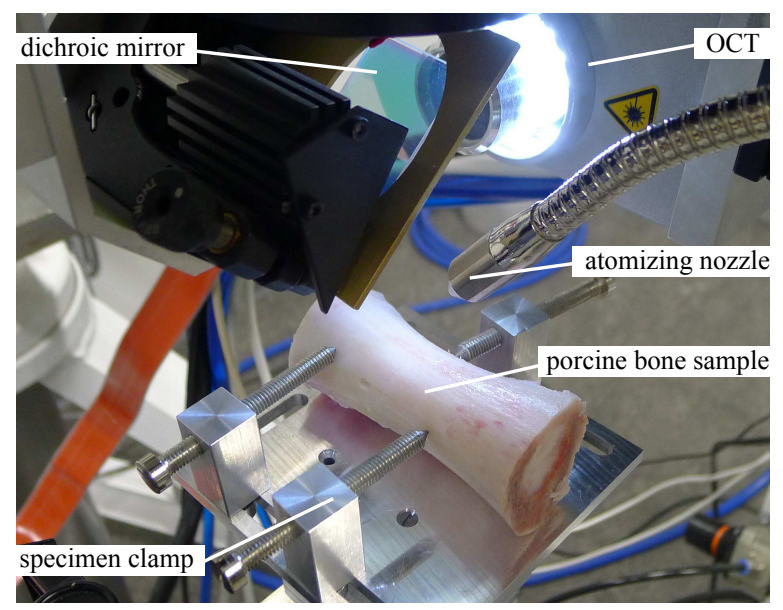

(b)

Figure 1: Experimental set-up for controlled laser ablation: (a) Components of the combined Er:YAG laser and OCT system with overlapping working volumes. Displacement of (CS) Laser $_{\text {and }}(\mathrm{CS})_{\text {OCT }}$ exaggerated for illustration purposes. (b) Composition in the laboratory for ablation on porcine bone sample with atomizing nozzle for moistening purposes.

\section{MATERIAL AND METHODS}

\subsection{Components and Experimental Set-Up}

The realized experimental set-up consists of two laser systems combined in an overlapping work space, as shown in Fig. 1(a). A pulsed Er:YAG laser beam (DPM 15 Laser Module, Pantec Engineering/3m.i.k.r.o.n ${ }^{\mathrm{TM}}$, Ruggell, Liechtenstein) is released onto the tissue by an electromechanical shutter. Subsequently, its course is deflected and modulated using mirrors and lenses adapting the beam geometry to following components. By passing through a focusing unit as well as an x-y-scanning system (hurrySCAN \& varioSCAN, SCANLAB, Puchheim, Germany) and finally by being focused by an f-theta flat-field lens a working volume of $10 \times 10 \times 10 \mathrm{~mm}^{3}$ is realized. The OCT (Ganymede, Thorlabs Inc, Newton, New Jersey, USA) with its center wavelength of $930 \mathrm{~nm}$ is customized to match the long focal distance $(\approx 100 \mathrm{~mm})$ of the cutting laser and positioned perpendicular to the Er:YAG laser beam. A dichroic mirror aligns both optical paths and creates an intersecting working volume $\left(10 \times 10 \times 3 \mathrm{~mm}^{3}\right)$. An additional moving stage $( \pm 5 \mathrm{~mm})$ compensates the difference in axial field of view and, thereby, ensures OCT visibility for each Er:YAG focus height.

The optical properties of the Er:YAG laser, i.e. its high absorption in hydrous material, explain the necessity of tissue moistening during processing. Therefore, a fine water spray is applied onto the specimen surface by an atomizing nozzle (see Fig. 1(b)). Fresh porcine bone samples are used for the control experiments, prepared directly after cutting, and held moist in a water basin. For reasons of hygiene, planar wooden samples are used for general testing and adjustments before the actual specimen trials. Prior to the following experiments, the position of the neutral $(z=0)$ focal plane of the Er:YAG laser in relation to the neutral OCT moving stage position has to be approximated in a preparatory step. Thereby, ablation patterns for all further steps such as the highly accurate registration, (see Chap. 2.2) can be analyzed by OCT surface scans.

An adjustable specimen clamp positions the plane wooden sample close to the expected distance of the focal plane as well as in the OCT volume. In order to define its exact position, the laser focus is moved in the $x$ - $z$-plane within the workspace boundaries from lowest to highest. The ablation result is measured using a corresponding OCT scan. The $z$-height of the smallest incision width and therefore, the smallest diameter of the focus spot defines the offset between both neutral $z$-positions and is used as reference for all following experiments. Due to a relatively long Rayleigh length of the focused Er:YAG beam satisfactory ablation is observed for $z$-variation of about $1 \mathrm{~mm}$. Thus, a more exact definition appears to be superfluous. OCT data itself can be calibrated for distortion correction using special structures and algorithms. ${ }^{10}$ 


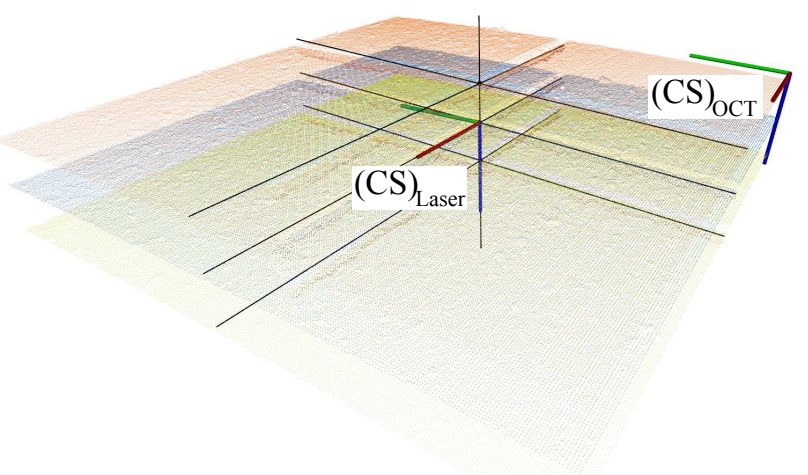

(a)

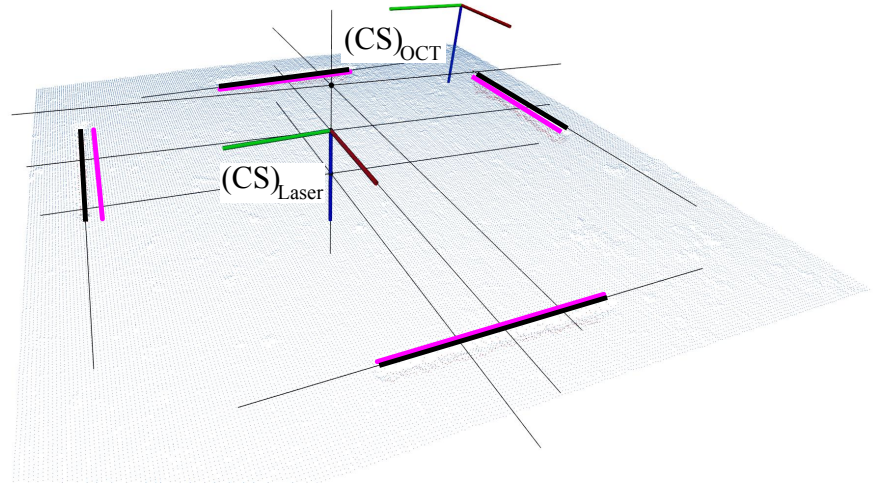

(b)

Figure 2: Representation of scanned surfaces for registration of laser and OCT coordinate systems $(x$ red, $y$ green, $z$ blue axis): (a) Segmented cross-like incision within three heights with reconstructed resulting (CS) Laser . (b) Analysis of scaling by comparing distances of planned (magenta) and actual (black) incisions.

\subsection{Registration of Er:YAG and OCT coordinate systems}

As seen in Fig. 1(a), the coordinate systems (CS) of Er:YAG laser and OCT do not correspond exactly after initial alignment of all components. For safe and depth controlled ablation results, a registration of (CS) Laser and $(\mathrm{CS})_{\mathrm{OCT}}$ has to be performed with higher precision than the already determined $z$-offset of coordinate origins. The computed transformation enables the user to plan cutting geometries directly in OCT image data. To identify this transformational relationship, a known registration structure is applied onto the planar wooden sample by the Er:YAG laser in its $x$ - $y$-plane. After automatically acquiring and transferring an OCT volume scan, the laser incision is detected within the data set by the planning software. The defined registration structure in form of a cross has its intersection point at the origin of (CS) Laser. This structure is applied and captured in three different height levels within the OCT volume on fresh areas of the wooden sample. Linear regression through each intersection point allows reconstructing the $z$-axis of $(\mathrm{CS})_{\text {Laser }}$ whereas $x$ - and $y$-axis are defined by the cross's lines (see Fig. 2(a)).

In addition to this tilt, commanded Er:YAG focus positions are subject to scaling effects, i.e. assigned scan movements do not result in estimated ablation patterns. This is expected to occur due to the number of optical components to be aligned and, therefore, an inaccurate impact of the laser beam on the scanning mirrors. To achieve the necessary precision for ablation process control, scaling between OCT and Er:YAG coordinate systems has to be calculated and considered in the transformation matrix. An additional set of laser incisions with well defined displacements is applied and analyzed. Actual and planned distances of the laser paths in $x$ and $y$ direction are compared in OCT data (see Fig. 2(b)) and scaling factors are determined.

\subsection{Iterative Control Algorithm}

Ablating a volume of hard tissue with smooth surfaces as well as high accuracy to size and causing a minimum harm to surrounding tissue, requires well chosen laser parameters for best laser tissue interaction. In preparation for specimen trials, several experimental series were conducted with varying pulse energy, frequency, and scanning speed. Ablation was analyzed with respect to resulting depth and width of incisions as well as tissue condition. Finally, multiple sets of parameters with discrete and specific ablation rates were defined in an extendible parameter list. This provides a simple and intuitive ablation model for the control algorithm and presents the basis for the laser energy to be selected. The adjustable scanning speed guarantees limited local exposure time within the ablation area.

The iterative steps of the control loop for automated hard tissue laser ablation are (i) OCT imaging and surface segmentation, (ii) model based path planning, and (iii) bone ablation along calculated corrective laser paths. They are performed sequentially and, in combination with tissue moistening, result in an iterative ablation process, schematically outlined in Fig. 3(a). The original unaltered tissue surface is derived from an 


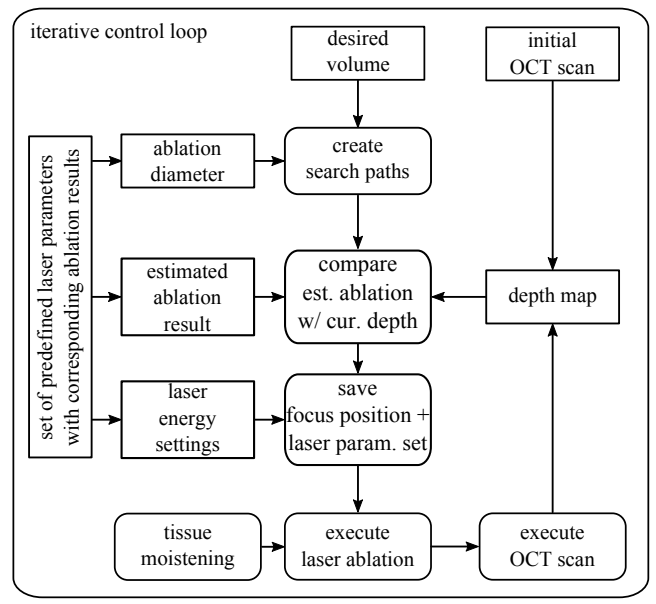

(a)

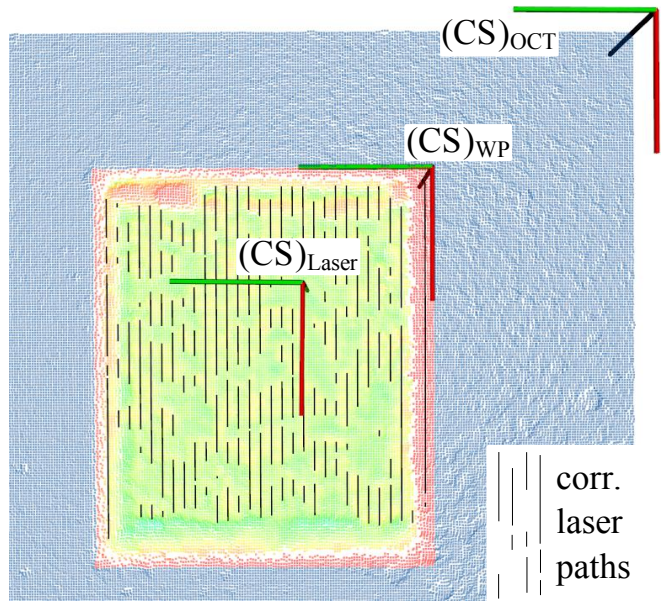

(b)

Figure 3: Ablation control process: (a) Iterative steps of the Er:YAG laser control loop using predefined sets of ablaiton parameters. (b) Planned corrective laser paths (black) for reaching desired ablation depth (green) within workpiece coordinate system (CS) $)_{\mathrm{WP}}(x$ red, $y$ green, $z$ blue axis).

initially acquired three-dimensional OCT scan. Raw data is automatically transferred and segmented employing a connected components algorithm and eventually, converted to a point cloud representing the surface. This visualization enables the user to set an arbitrary target geometry onto the tissue surface with defined dimensions. Additionally, a workpiece coordinate system (CS) WP is established (see Fig. 3(b)). The definition of (CS) WP facilitates a generic development of the control algorithm independent of target position in the working volume and complies with the modularity of the developed software architecture.

In addition to the target geometry, the user defines a desired depth of the incision. The algorithm generates a grid of possible laser paths on the target surface, which is adapted employing the chosen parameter set, i.e. its spacing is varied according to the expected ablation width. Based on a local altitude analysis within the target area, a subset of this grid is defined as corrective laser execution paths for each set of laser parameters by the planning algorithm. Parameter selection depends on the amount of remaining tissue. Height of the laser focus is adapted dynamically according to surface condition. The resulting combination of paths and individual laser parameters from the ablation rate model (see Fig. 3(b)) is executed by Er:YAG laser controller and scanning system. The ablation process is followed by moistening of the tissue and an OCT scan for the next iteration step. These ablation cycles approximate the demanded target geometry until no further corrective laser paths can be set by the algorithm, i.e. the achieved depth is within the tolerances of the parameter set with the smallest ablation rate. Since the algorithm works solely based on straight laser paths, it rotates the moving direction of the Er:YAG focus by $90^{\circ}$ with each ablation cycle. Due to the not deterministic beam release of the pulsed laser source in combination with the shutter ablation geometry tends to be slightly ragged at starting and end points of laser paths. Rotation minimizes this effect and creates sharp edges.

\section{RESULTS}

Developed registration and ablation control processes are examined for accuracy and reproducibility and, therefore, experiments on both procedures were executed repeatedly $(n=5)$. All results are summarized in Tab. 1 . The robustness of the presented, highly automated registration process for (CS) Laser and (CS) OCT is verified by small standard deviation of calculated tilt and offset. For example, adjustment values in x-direction result in a rotation of $\alpha=2.5^{\circ} \pm 0.1^{\circ}$ and a translation of $\Delta x=3.71 \mathrm{~mm} \pm 0.025 \mathrm{~mm}$. The scaling factor is determined to $s_{x}=0.9863 \pm 0.0003$.

The functionality of the iterative control algorithm is assessed by analyzing an applied geometry of defined dimensions $\left(3 \times 3 \times 1 \mathrm{~mm}^{3}\right)$ in porcine bone specimen. Condition of the processed tissue is evaluated by sight, whereas geometrical accuracy is externally measured using a high precision measuring arm (FARO Gage, 
Florida, USA). Results show an achieved depth of $z=0.96 \mathrm{~mm} \pm 0.081 \mathrm{~mm}$ which lies within tolerances defined by the parameter set with the least possible laser energy. This set exhibits a minimal expected ablation rate of $d_{\min }=100 \mu \mathrm{m}$ and width of $w_{\min }=200 \mu \mathrm{m}$ per process cycle. Maximum values are $d_{\max }=600 \mu \mathrm{m}$ and $w_{\max }=300 \mu \mathrm{m}$. Length and width result to $\ell_{\text {length }}=2.87 \mathrm{~mm} \pm 0.035 \mathrm{~mm}$ and $\ell_{\text {width }}=2.82 \mathrm{~mm} \pm 0.047 \mathrm{~mm}$, respectively. The shortening of both sides is exhibited due to the conical beam shape and resulting conical ablation, which is measured by the measuring tip at the ablation surface.

Table 1: Results for repeated registration of (CS) $)_{\text {Laser }}$ and (CS) OCT and for OCT controlled laser ablation of porcine bone samples

\begin{tabular}{|l|l|r|r|}
\hline process step & measured dimension & mean value & standard deviation \\
\hline \hline registration & rotation $\alpha$ & $2.5^{\circ}$ & $\pm 0,118^{\circ}$ \\
& rotation $\beta$ & $-0.37^{\circ}$ & $\pm 0,085^{\circ}$ \\
& rotation $\gamma$ & $0.75^{\circ}$ & $\pm 0,25^{\circ}$ \\
\cline { 2 - 4 } & translation $\Delta x$ & $3.71 \mathrm{~mm}$ & $\pm 25 \mu \mathrm{m}$ \\
& translation $\Delta y$ & $4.94 \mathrm{~mm}$ & $\pm 10 \mu \mathrm{m}$ \\
& translation $\Delta z$ & $1.1 \mathrm{~mm}$ & $\pm 230 \mu \mathrm{m}$ \\
\cline { 2 - 4 } & scaling factor $s_{x}$ & 0.9863 & $\pm 0,0003$ \\
& scaling factor $s_{y}$ & 0.9497 & $\pm 0,0004$ \\
\hline \multirow{2}{*}{ controlled ablation } & depth & $0.96 \mathrm{~mm}$ & $\pm 81 \mu \mathrm{m}$ \\
& width $\ell_{\text {width }}$ & $2.87 \mathrm{~mm}$ & $\pm 35 \mu \mathrm{m}$ \\
& length $\ell_{\text {length }}$ & $2.82 \mathrm{~mm}$ & $\pm 47 \mu \mathrm{m}$ \\
\hline
\end{tabular}

To assess the hard tissue surface after a finished controlled ablation process Fig. 4(a) and Fig. 4(b) show the user visualization and the rendered OCT image of a target geometry $\left(4 \times 2 \times 1.5 \mathrm{~mm}^{3}\right)$. Sharp and defined edges with slightly conical characteristics are demonstrated. The bottom surface of the target area shows a rippled structure due to the heterogeneous bone material, but stays within the tolerances defined by the minimal ablation parameter set. All results are obtained with minimum harm to tissue and no visible signs of overheating (see Fig. 4(c)). Each iteration cycle takes less than $20 \mathrm{~s}$ due to the fast segmentation algorithm and automatic data transfer, resulting in a total processing time of about $240 \mathrm{~s}$. Due to the short iteration cycle, drying effects in the surface tissue are minimized and optimal laser tissue interaction is realized with a small water spray rate ( $7 \mathrm{\mu l}$ per spray pulse) onto an area of $40 \mathrm{~mm}^{2}$.

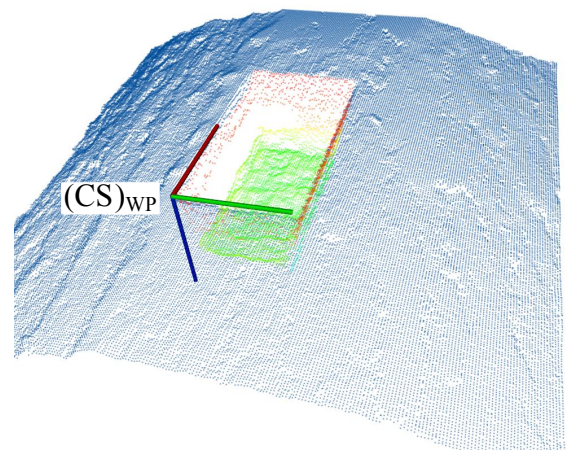

(a)

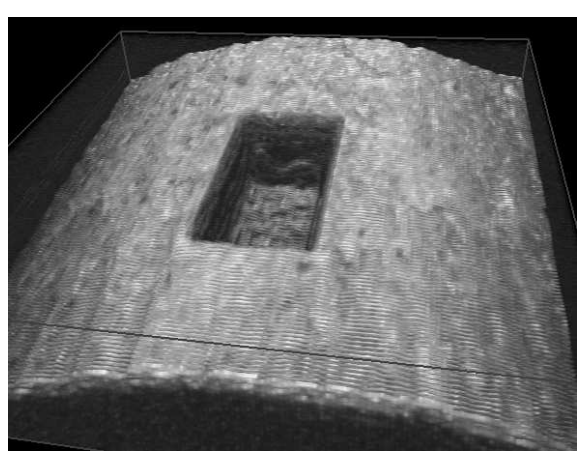

(b)

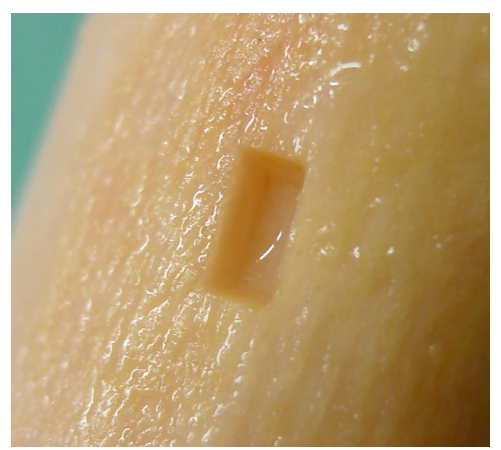

(c)

Figure 4: Different representations of ablation results: (a) Point cloud visualization of achieved desired depth (green). (b) OCT image of the target geometry $\left(4 \times 2 \times 1.5 \mathrm{~mm}^{3}\right)$. (c) Bone surface after controlled ablation without visual signs of necrosis. 


\section{CONCLUSIONS}

The presented system and concept provide a suitable solution for controlled hard tissue ablation. Automatic image data computing and transfer as well as taking into account the requirements of tissue laser interaction guarantee high accuracy and fast results. A safe ablation towards critical structures at a desired depth can be provided and enables new opportunities in laser surgery. To the best of our knowledge, this is the fastest OCT controlled laser ablation procedure with the highest degree of automation realized to date.

Future work will aim at dynamically adapting the ablation rates in the control model parameter sets to handle tissues with a high degree of heterogeneity. Combination with RGB information of stereo camera data is a desired aim as well, when considering usability in the operating room, since it would provide a more intuitive user interaction. Finally, the potential of OCT imaging lies in the ability to view underlying structures, e.g. bone thickness, as mentioned above. ${ }^{8}$ This still poses the major challenge in integrating a robust segmentation of the second boundary layer. Thereafter, our control loop could be closed using the remaining tissue thickness as control variable.

\section{REFERENCES}

[1] Cloutier, M., Girard, B., Peel, S. A., Wilson, D., Sandor, G. K., Clokie, C. M., and Miller, D., "Calvarial bone wound healing: a comparison between carbide and diamond drills, Er:YAG and femtosecond lasers with or without BMP-7," Oral Surgery, Oral Medicine, Oral Pathology, Oral Radiology, and Endodontology 110(6), 720-728 (2010).

[2] Panduric, D. G., Bago, I., Katanec, D., Zabkar, J., Ivana, M., and Anic, I., "Comparison of Er:YAG laser and surgical drill for osteotomy in oral surgery: An experimental study," Journal of Oral and Maxillofacial Surgery 70(11), 2515-2521 (2012).

[3] Pourzarandian, A., Watanabe, H., Aoki, A., Ichinose, S., Sasaki, K. M., Nitta, H., and Ishikawa, I., "Histological and TEM examination of early stages of bone healing after Er:YAG laser irradiation," Photomedicine and Laser Surgery 22(4), 342-350 (2004).

[4] de Mello, E., Pagnoncelli, R., Munin, E., Filho, M., de Mello, G., Arisawa, E., and de Oliveira, M., "Comparative histological analysis of bone healing of standardized bone defects performed with the Er:YAG laser and steel burs," Lasers in Medical Science 23(3), 253-260 (2008).

[5] Stopp, S., Svejdar, D., von Kienlin, E., Deppe, H., and Lueth, T. C., "A new approach for creating defined geometries by navigated laser ablation based on volumetric 3-D data," IEEE Transactions on Biomedical Engineering 55(7), 1872-80 (2008).

[6] Kahrs, L. A., Raczkowsky, J., Werner, M., Knapp, F. B., Mehrwald, M., Hering, P., Schipper, J., Klenzner, T., and Wörn, H., "Visual servoing of a laser ablation based cochleostomy," Proc. SPIE 6918(69182C), $1-11(2008)$.

[7] Leung, B. Y., Webster, P. J., Fraser, J. M., and Yang, V. X., "Real-time guidance of thermal and ultrashort pulsed laser ablation in hard tissue using inline coherent imaging," Lasers in Surgery and Medicine 44(3), 249-256 (2012).

[8] Zhang, Y., Pfeiffer, T., Weller, M., Wieser, W., Huber, R., Raczkowsky, J., Schipper, J., Wörn, H., and Klenzner, T., "Optical coherence tomography guided laser cochleostomy: Towards the accuracy on tens of micrometer scale," BioMed Research International 251814, 1-10 (2014).

[9] Fuchs, A., Schultz, M., Krüger, A., Kundrat, D., Diaz Diaz, J., and Ortmaier, T., "Online measurement and evaluation of the Er:YAG laser ablation process using an integrated OCT system," Biomedizinische Technik 57(1), 434-437 (2012).

[10] Díaz Díaz, J., Rahlves, M., Majdani, O., Reithmeier, E., and Ortmaier, T., "A one step vs. a multi step geometric calibration of an optical coherence tomography," Proc. SPIE 8573(85730D), 1-12 (2013). 